\title{
Approach to Brain Magnetic Resonance Imaging for Non-Radiologists
}

\author{
Amir Taree ${ }^{a}$, Vahid Eslami ${ }^{b}$, Sahra Emamzadehfard ${ }^{c, d}$
}

\begin{abstract}
The goal of this review is to provide a guide to magnetic resonance imaging (MRI) reading for non-radiologists. A thorough literature search was conducted using the keywords "MRI", "CT", "Non-radiologist" and "MRI interpretation" to develop an approach to MRI reading for non-radiologists. Common indications for a brain MRI include workup of an intracranial tumor, chronic headache, seizure disorder, and confirmation of a stroke. When assessing for an intracranial tumor, MRI is the preferred diagnostic modality. Computed tomography (CT) has much lower resolution and is typically reserved for the emergency setting. T1 weighted images provide anatomically relevant images of the brain parenchyma that will be familiar to nonradiologists. In contrast to T1 weighted images, fluid is bright in T2 and white matter will appear darker than gray matter. Fluid attenuation inversion recovery (FLAIR) is most sensitive for edema and parenchymal abnormalities like a low-grade glioma. The main purpose of diffusion-weighted imaging (DWI) and apparent diffusion coefficient (ADC) sequences are to visualize acute ischemic stroke. Although non-radiologists generally have a greater exposure to head CT images, the same foundational principles of CT head interpretation can apply to brain MRI reading. Benefits of brain imaging by MRI includes obtaining a multi-planar assessment of the brain, highly detailed images of the brain, and using different MRI sequences to assess for different pathology.
\end{abstract}

Keywords: MRI; Non-radiologist; CT

\section{Introduction}

Interpreting brain magnetic resonance imaging (MRI) as a

Manuscript submitted August 19, 2020, accepted August 28, 2020

Published online September 4, 2020

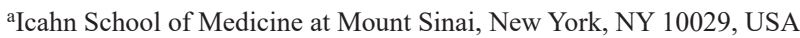

bDepartment of Neurology, University of Texas Health Science Center at San Antonio, San Antonio, TX 78229, USA

'Department of Radiology, University of Texas Health Science Center at San Antonio, San Antonio, TX 78229, USA

${ }^{\mathrm{d} C o r r e s p o n d i n g ~ A u t h o r: ~ S a h r a ~ E m a m z a d e h f a r d, ~ D e p a r t m e n t ~ o f ~ R a d i o l o g y, ~}$ University of Texas Health Science Center at San Antonio, San Antonio, TX 78229, USA. Email: Sahra.e.fard@gmail.com

doi: https://doi.org/10.14740/jnr628 non-radiologist can often be intimidating at first glance due to the various considerations of sequences and parameters that must be made. Although non-radiologists generally have a greater exposure to head computed tomography (CT) images, the same foundational principles of CT head interpretation can apply to brain MRI reading [1]. With an appreciation of the basic normal anatomical structures, each MRI sequence can be assessed for how it changes the appearance of normal structures and abnormal findings. The goal of this review is to provide a systematic approach to brain MRI interpretation for non-radiologists. We will discuss the indications for a brain MRI, special considerations for non-radiologists, types of MRI sequences, and manifestations of common pathophysiology on brain MRI.

\section{Indications for a Brain MRI}

Common indications for a brain MRI include workup of an intracranial tumor, chronic headache, seizure disorder, and confirmation of a stroke.

When assessing for an intracranial tumor, MRI is the preferred diagnostic modality as CT has much lower resolution and is typically reserved for the emergency setting. MRI is more specific and sensitive for abnormalities within the brain itself due to providing a much more detailed depiction of the anatomy [2]. Chronic headache is another indication for a brain MRI. Chronic refers to a headache frequency of 15 or more days for longer than 3 months in the absence of an organic pathology. In patients with new daily persistent headache, hemicrania continua, cluster headache, and paroxysmal hemicrania, imaging is indicated to exclude a secondary cause of the headache. However, it should be noted that MRI findings identify a treatable cause of chronic headache in $<1 \%$ of cases [3]. In the evaluation of a first seizure in children and adults, MRI is useful to detect any structural abnormalities that can cause seizure disorders. This can include, but is not limited to infarcts, tumors, medial temporal lobe sclerosis, and cortical dysplasia. Finally, confirmation of a stroke is another common indication for a brain MRI. At many centers due to its widespread availability and faster scan times, non-contrast $\mathrm{CT}$ is the preferred imaging method for initial evaluation of a stroke, especially for intracranial hemorrhage. However, MRI with diffuse-weighted imaging is superior to non-contrast CT for earlier detection of acute ischemia and excluding stroke mimics. In one study of 135 patients with acute stroke who are screened for intravenous thrombolytic therapy, patients ini- 

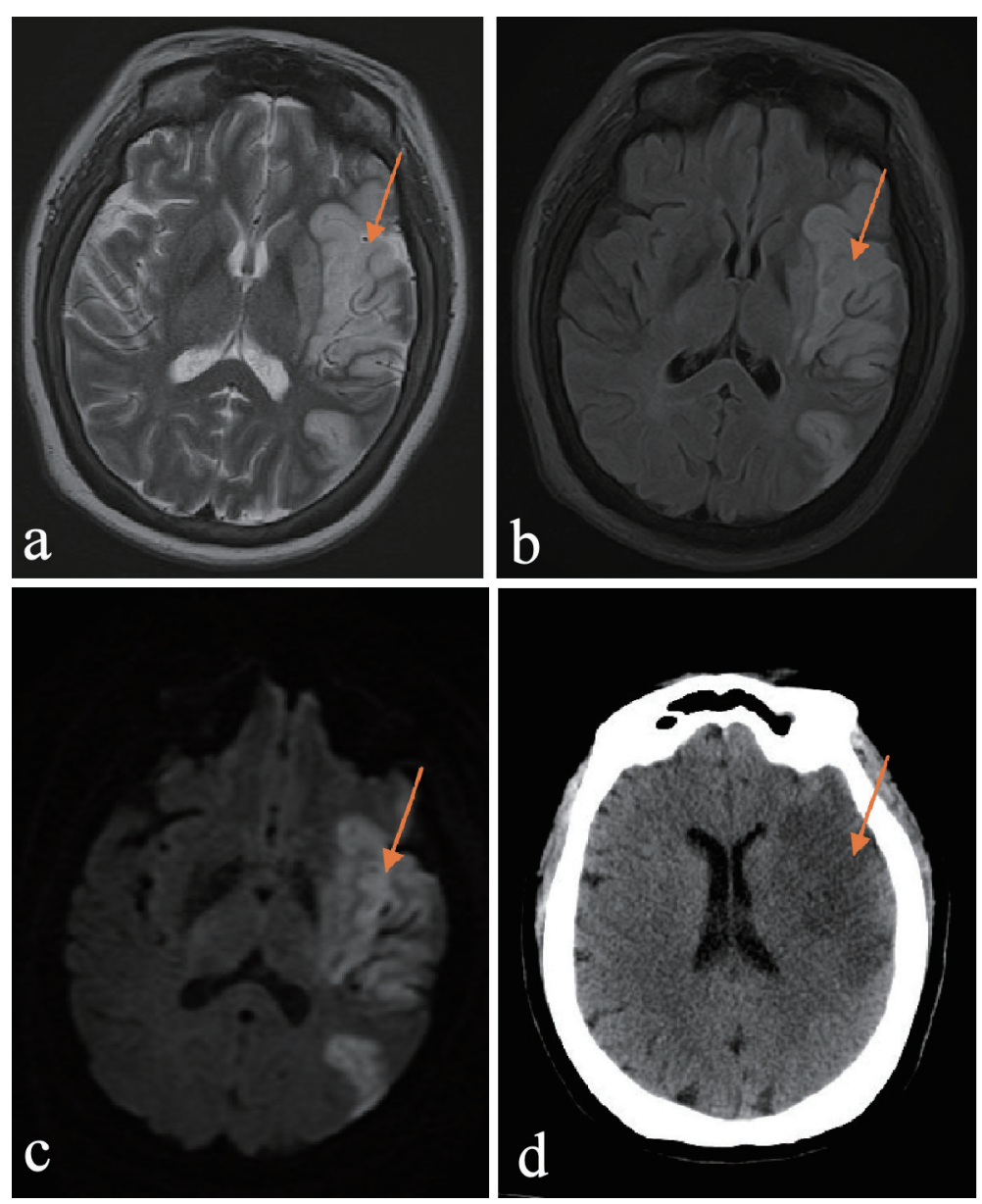

Figure 1. (a) T2 image of left MCA territory infarct, with associated vasogenic edema. (b) FLAIR image of left MCA territory infarct, with associated vasogenic edema. (c) DWI image of left MCA territory infarct, with associated vasogenic edema. (d) CT image of left MCA territory infarct, with associated vasogenic edema. MCA: middle cerebral artery; FLAIR: fluid attenuation inversion recovery; DWI: diffusion-weighted imaging; CT: computed tomography.

tially screened by MRI led to reduced door-to-needle times of $\leq 60 \min [4]$.

\section{Considerations for Non-Radiologists}

There are various benefits and limitations for brain MRI that non-radiologists must consider. Benefits of brain imaging by MRI includes obtaining a multi-planar assessment of the brain, highly-detailed images of the brain, and using different MRI sequences to assess for different pathology [5]. Another major benefit of MRI is in children who are not exposed to any ionized radiation with MRI compared to CT imaging. However, there are several limitations as well. MRIs are not nearly as available compared to $\mathrm{CT}$ imaging at many centers. It requires a much longer investigation compared to $\mathrm{CT}$ and other imaging modalities, which can create problems for patients who may be claustrophobic. Due to their greater availability, patients have much shorter waiting times for CT imaging than MRI. Finally, there are several contraindications to obtaining a MRI such as metallic implants like pacemakers.

\section{MRI Sequences}

We will discuss a brief overview of the different sequences and images that can be seen with MRI using different pulses and signals. Whereas in CT imaging, the focus is on comparing the density of images, in MRI images are described by signal intensity. "Low signal intensity" images will appear dark while "high signal intensity" appears bright.

T1 weighted images provide anatomically relevant images of the brain parenchyma that will be familiar to non-radiologists [5]. Any fluid such as cerebrospinal fluid in the ventricles or fluid in the orbits will appear dark. Air is nearly black. Gray matter will appear darker than white matter.

T2 weighted images are another standard sequence. In contrast to T1 weighted images, fluid is bright in $\mathrm{T} 2$ and white matter will appear darker than gray matter.

Fluid attenuation inversion recovery (FLAIR) is another commonly used sequence. It appears similar to T2, but fluid is even darker. FLAIR is useful in identifying edema and inflammation. It can be useful in depicting the periventricular plaques that are indicative of multiple sclerosis. 

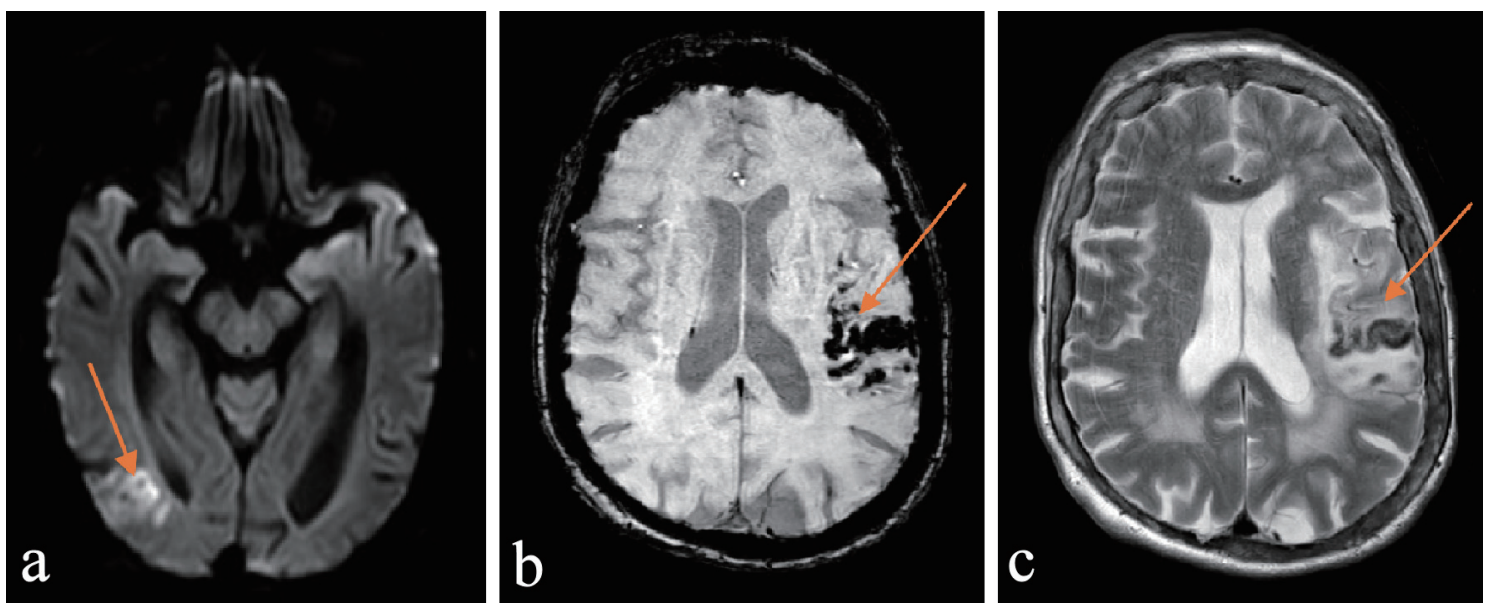

Figure 2. (a) DWI image of ischemic stroke with hemorrhagic transformation. (b) SWI image of ischemic stroke with hemorrhagic transformation. (c) T2 image of ischemic stroke with hemorrhagic transformation. DWI: diffusion-weighted imaging; SWI: susceptibility-weighted imaging.

Finally, diffusion-weighted imaging (DWI) and apparent diffusion coefficient (ADC) images show the movement of water in the brain. By describing the diffusion of water throughout the brain, findings such as stroke, abscesses, and tumors can be identified.

\section{Manifestations of Common Pathophysiology on Brain MRI}

In $\mathrm{T} 1$ weighted images, tumors will appear dark with low signal intensity. Gadolinium contrast can be added to the T1 which will make a tumor or abscess appear bright. If a lesion has dark signal on T1, but bright signal on gadolinium contrast, it is "contrast enhancing" [6]. If only the edges are enhanced, it is a "ring enhancing" lesion such as a brain abscess [7].

T2 weighted images are more useful to evaluate cerebrospinal fluid (CSF) which appears bright white while the brain parenchyma appears light gray. T2 sequence can be used to identify bright signal of edema around tumors, subacute stroke, or hemorrhage. In addition, it can be used to assess the size and symmetry of the ventricles as well as identify periventricular plaques or white matter changes in multiple sclerosis where demyelination appears as a bright signal in the white matter [7].

FLAIR is most sensitive for edema and parenchymal abnormalities like a low-grade glioma. Figure 1a-c compare MRI images of left middle cerebral artery (MCA) territory infarct, with associated vasogenic edema compared to the same pathology on CT (Fig. 1d).

As discussed earlier, the main purpose of DWI and ADC sequences are to visualize acute ischemic stroke. In a patient with stroke symptoms who has a CT performed that shows no signs of hemorrhage, the DWI will show the stroke as a bright signal while ADC will show the stroke as a dark signal. The difference between ischemic strokes with hemorrhagic transformation can be seen in MRI compared to CT (Figs. 2, 3).

While CT is the preferred imaging modality for hemorrhage, MRI is the study of choice for brain tumors, multiple
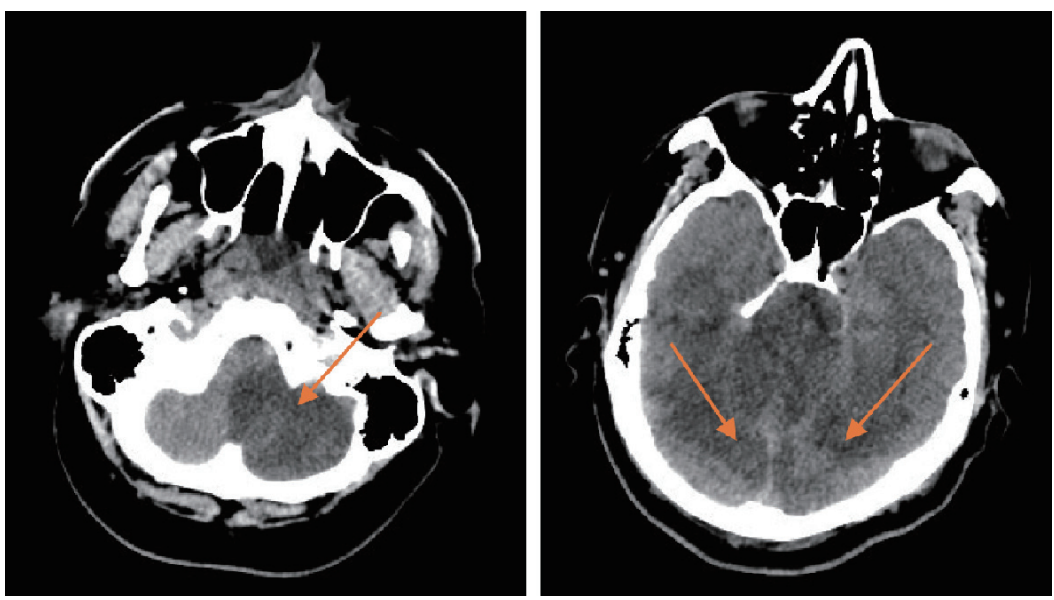

Figure 3. CT image of acute infarct in cerebellum and occipital lobe. CT: computed tomography. 
sclerosis and ischemic stroke [6].

\section{Acknowledgments}

Images courtesy of Dr. Ali Seifi's archives.

\section{Financial Disclosure}

None to declare.

\section{Conflict of Interest}

None to declare.

\section{Author Contributions}

Amir Taree conducted literature review. Amir Taree, Sahra Emamzadehfard, and Vahid Eslami contributed to the writing of the manuscript.

\section{Data Availability}

The authors declare that data supporting the findings of this study are available within the article.

\section{References}

1. Approach to MRI brain. LearningNeurology.com. https:// learningneurology.com/diagnostic-tests/approach-to-mribrain/. Published October 14, 2018. Accessed August 3, 2020.

2. Hess CP. Exploring the brain: is ct or mri better for brain imaging? UCSF Radiology. https://radiology.ucsf.edu/ blog/neuroradiology/exploring-the-brain-is-ct-or-mribetter-for-brain-imaging. Published November 16, 2015. Accessed August 3, 2020.

3. Frishberg BM. The utility of neuroimaging in the evaluation of headache in patients with normal neurologic examinations. Neurology. 1994;44(7):1191-1197.

4. Shah S, Luby M, Poole K, Morella T, Keller E, Benson RT, Lynch JK, et al. Screening with MRI for Accurate and Rapid Stroke Treatment: SMART. Neurology. 2015;84(24):2438-2444.

5. Smith D. MRI brain (summary): radiology reference article. Radiopaedia Blog RSS. https://radiopaedia.org/articles/mri-brain-summary?lang=us. Accessed August 3, 2020.

6. Anvekar DB. Ischemic stroke and vascular territories of brain. http://www.neuroradiologycases.com/2012/09/ ischemic-stroke-and-vascular.html. Accessed August 5, 2020.

7. Brain MRI: a systematic reading. Neurosurgery Basics. https://neurosurgerybasics.com/brain/brain-mri-a-systematic-reading/. Published June 11, 2020. Accessed August $3,2020$. 